\title{
Strain rate effects on mechanical properties in tension of aluminium alloys used in armour applications
}

\author{
E. Cadoni ${ }^{1}$, M. Dotta ${ }^{1}$, D. Forni ${ }^{1}$, S. Bianchi ${ }^{2}$, and H. Kaufmann ${ }^{2}$ \\ ${ }^{1}$ DynaMat Lab., University of Applied Sciences of Southern Switzerland, 6952 Canobbio, Switzerland \\ ${ }^{2}$ RUAG Defence, RUAG Schweiz AG, 3602 Thun, Switzerland
}

\begin{abstract}
The mechanical properties in tension of two aluminium alloys (AA5059-H131 and AA7039-T651) used in armour applications were determined from tests carried out over a wide range of strain-rates on round specimens. The experimental research was developed in the DynaMat laboratory of the University of Applied Sciences of Southern Switzerland. The target strain rates were set at the following four levels: $10^{-3}, 30,300$ and $1000 \mathrm{~s}^{-1}$. The quasi-static tests were performed with a universal electromechanical machine, whereas a hydro-pneumatic machine and a Split Hopkinson Tensile Bar apparatus were used for medium and high strain-rates respectively. The required parameters by the Johnson-Cook constitutive law were also determined.
\end{abstract}

\section{Introduction}

The use of aluminium alloys in the construction of defence vehicles is strongly increasing in these last decades because they require new lightweight armours for improving their survivability without sacrificing efficiency and performance.

Many researchers reported the alloy composition, strainrate, microstructure and service temperature may have an effect on mechanical properties and failure mechanism of aluminium alloys [1,2]. Naka et al. [3] measured the strainrate sensitivity $\left(5 \cdot 10-5 \div 50 \mathrm{~s}^{-1}\right)$ of a $5 \mathrm{xxx}$ aluminium alloy at $20-250{ }^{\circ} \mathrm{C}$. A negative strain-rate sensitivity was obtained at room temperature. Pérez-Bergquist et al. [4] studied the behaviour of 5059 and 7039 aluminium alloys in compression and shear in both quasi-static and dynamic strain-rates regimes.

Thus, the objective of this work is the examination of the strain-rate effect on the mechanical properties in tension of 5059-H131 and 7039-T651 aluminium alloys.

\section{Materials}

The first analysed material is the AA 5059-H131 that is a magnesium $(\mathrm{Mg})$ based non heat treatable alloy that are strengthened by mechanical strain hardening. The strain hardening process results in the $5 \mathrm{xxx}$ series alloy gives the " $H$ " designation instead of the " $T$ " designation that is typical for heat treatable alloys. On the other hand, the "H131" tag indicates that during the production process the material was strengthened by mechanical strain hardening.

The second material is part of the $7 \mathrm{xxx}$ series zinc $(\mathrm{Zn})$ based aluminium alloy: AA 7039-T651. The "T651" tag indicates the temper designation, which is obtained from a solution heat treated and artificially aged to a stable condition.

The chemical compositions of the considered materials are shown in the following table 1.
Table 1. Chemical composition.

\begin{tabular}{|c|c|c|}
\hline & AA 5059-H131 & AA7039-T651 \\
\hline $\mathrm{Si}$ & 0,06 & 0,15 \\
\hline $\mathrm{Fe}$ & $<0,01$ & 0,12 \\
\hline $\mathrm{Cu}$ & 0,84 & 0,06 \\
\hline $\mathrm{Mg}$ & 5,04 & 2,44 \\
\hline $\mathrm{Zn}$ & 0,56 & 4,31 \\
\hline $\mathrm{Cr}$ & $<0,01$ & 0,18 \\
\hline $\mathrm{Ti}$ & 0,02 & 0,02 \\
\hline $\mathrm{Al}$ & Balance & Balance \\
\hline
\end{tabular}

\subsection{Specimens}

Round samples having $3 \mathrm{~mm}$ in diameter and $5 \mathrm{~mm}$ of gauge length have been used (see figure 1).

In order to measure the fracture parameters on the specimens surface, the gauge length of $5 \mathrm{~mm}$ has been marked before the test.

The specimens analysis has been carried out studying both the experimental results in terms of engineering and true stress versus strain curves and in terms of fracture failure.

The characteristics of fracture as the reduced area of the specimen cross section after failure in the necking zone as well as the fracture strain have been obtained by means of two images taken before and after the test of each specimen. In order to do this, the specimen is at best reconstructed by bringing together the two broken parts, so that both the diameter and the meridional radius of curvature at the reduced section could be measured.

\section{Experimental set-up}

Tensile testing at several strain-rates has been performed using different experimental set-ups [5].

All tests were performed at room temperature, and the target strain-rates were set at the following four levels: $10^{-3}, 30,300$ and $1000 \mathrm{~s}^{-1}$. 


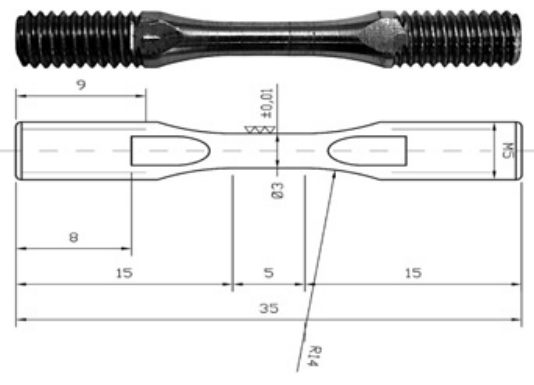

Fig. 1. Specimen geometry.

Table 2. Experimental test planning.

\begin{tabular}{|c|c|c|c|c|}
\hline \multirow{2}{*}{} & \multicolumn{4}{|c|}{ Strain-rates $\left[\mathrm{s}^{-1}\right]$} \\
\cline { 2 - 5 } & $10^{-3}$ & 30 & 300 & 1000 \\
\hline AA 5059-H131 & 1 & 3 & 3 & 3 \\
\hline AA 7039-T651 & 1 & 3 & 3 & 3 \\
\hline
\end{tabular}

Three tensile tests were conducted for each strain-rate, except for quasi-static condition where only one test was executed. In the following table 2, the experimental test planning, with the number of tested samples is reported.

\subsection{Quasi-static tests}

Quasi-static tests have been performed by means of a universal electromechanical testing machine Zwick/Roell $-\mathrm{Z} 50$ which has the maximum load bearing capacity of $50 \mathrm{kN}$.

\subsection{Intermediate strain-rate}

Intermediate strain-rate behaviour in tension has been investigated by means of a hydro-pneumatic machine (HPM), whose scheme is reported in figure 2.

The functioning of the machine is the following:

- Firstly, the water is filled in the upper tank-chamber and the gas is filled in the lower tank-chamber. At this moment, equal pressure is established in both chambers so that the forces acting on the two piston faces are in equilibrium.

- Secondly, the specimen is fixed to both the piston shaft and the elastic bar instrumented with a strain gauge.

- Lastly, by activating a fast electro-valve closing the water chamber, the force exerted by the gas pressure on one face of the piston prevails, accelerating the piston, which simultaneously loads the specimen and pushes water to flow out through the calibrated orifice at a constant speed, with the result of imposing a strain to the specimen with a constant strain-rate.

The stress measurements were obtained by the strain gauge of the elastic bar connected to the specimen while the strain measurements were obtained by means of two contactless displacement transducers placed at the ends of the specimen.

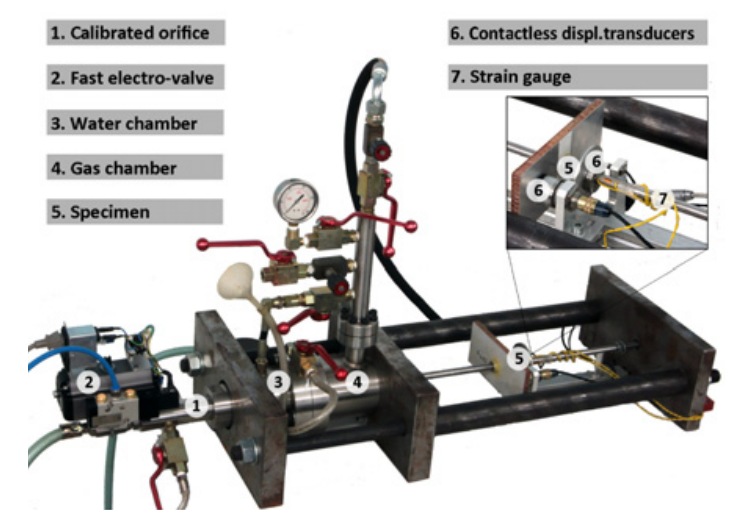

Fig. 2. Hydro-Pneumatic Machine.

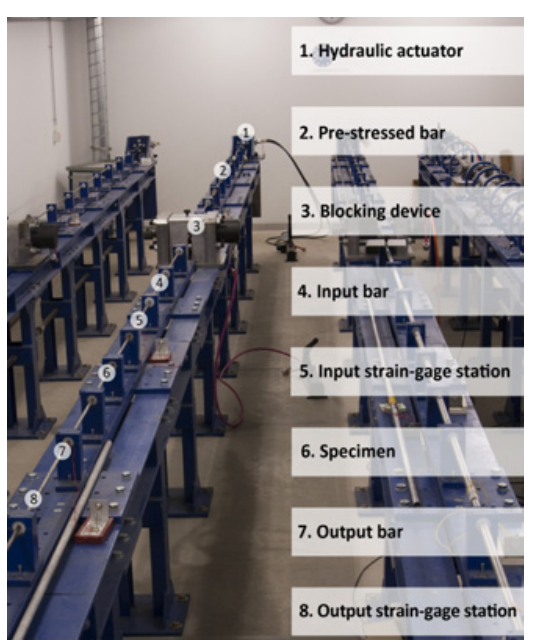

Fig. 3. JRC-split Hopkinson tensile bar.

\subsection{High strain rate}

The high strain-rate tests have been performed using a Split Hopkinson Tensile Bar [6] that consists of two cylindrical high strength steel bars, having a diameter of $10 \mathrm{~mm}$, with a length of respectively 9 and 6 meters for the input and the output bar, as represented in figure 3.

The aluminium specimen is assembled between the two bars. Input and output bars were instrumented with strain gauges which measure the incident, the reflected and the transmitted pulses acting on the specimen cross section.

The present set-up has the following conditions: i) the bar diameter $(10 \mathrm{~mm})$ is small in comparison with the pulse length $(12 \mathrm{~m})$; ii) the specimen length is short so that the time taken by the wave to propagate through the specimen is short compared to the total time of the test. These conditions allow many reflections inside the specimen necessary for reaching a homogeneous stress and strain distribution along the specimen gauge length, what means also equilibrium of the forces acting on both ends of the specimen.

Having fulfilled the conditions i) and ii) and being the two bars elastically loaded the one-dimensional elastic plane stress wave propagation theory can be applied to the input bar-specimen-output bar system as it is extensively 


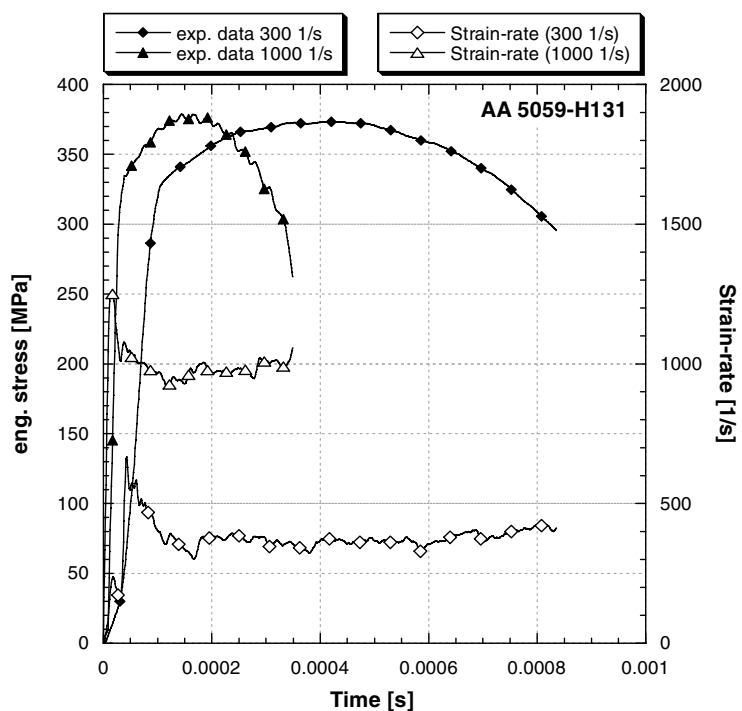

Fig. 4. Engineering stress and strain-rate versus time @ $300 \mathrm{~s}^{-1}$ and $1000 \mathrm{~s}^{-1}$ for AA 5059-H131.

shown in $[5,6]$. In these papers the three relationships are proofed which allow to calculate the stress, the strain and the strain rate in the specimen material versus time from the records of the incident $\left(\varepsilon_{I}\right)$, the reflected $\left(\varepsilon_{R}\right)$ and the transmitted $\left(\varepsilon_{T}\right)$ pulses.

$$
\begin{aligned}
\sigma_{E}(t) & =E_{0} \frac{A_{0}}{A} \varepsilon_{T}(t) \\
\varepsilon_{E}(t) & =-\frac{2 C_{0}}{L} \int_{0}^{t} \varepsilon_{R}(\tau) d \tau \\
\dot{\varepsilon}(t) & =-\frac{2 C_{0}}{L} \varepsilon_{R}(t)
\end{aligned}
$$

where, $E_{0}$ is the bars elastic modulus, $A_{0}$ is the bars crosssectional area, $A$ is the specimens cross-sectional area, $L$ is the specimen gauge length and $C_{0}$ is the sound velocity of the bar material.

Using the equation (3) and observing figures 4 and 5 (where stress and strain-rate versus time are shown) it can be noted that during the plastic zone the strain-rate is nearby constant.

\section{Results}

Representative engineering and true stress versus strain curves at different strain-rates for the analysed alloys are depicted in the following figures 6-9.

A better and brief representation of the strain-rate sensitivity of these alloys can be obtained by examination of the trends of the yield strength and the ultimate tensile strength (at the onset of necking) as a function of strainrate (see figure 10). In the same plots also the uniform and fracture strain and the reduction in cross-sectional area are reported. For each of these trends, a visual trend line is included to help distinguish the data sets.

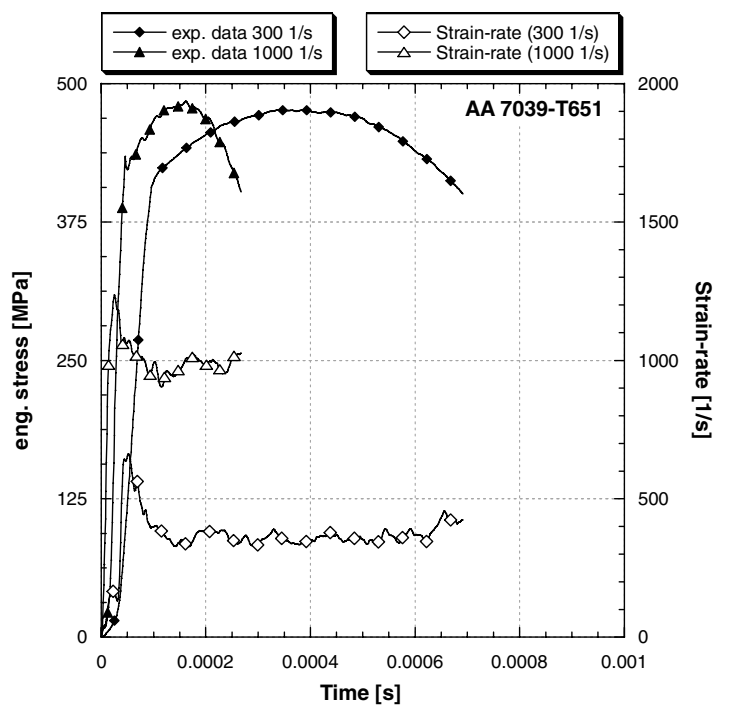

Fig. 5. Engineering stress and strain-rate versus time @ $300 \mathrm{~s}^{-1}$ and $1000 \mathrm{~s}^{-1}$ for AA 7039-T651.

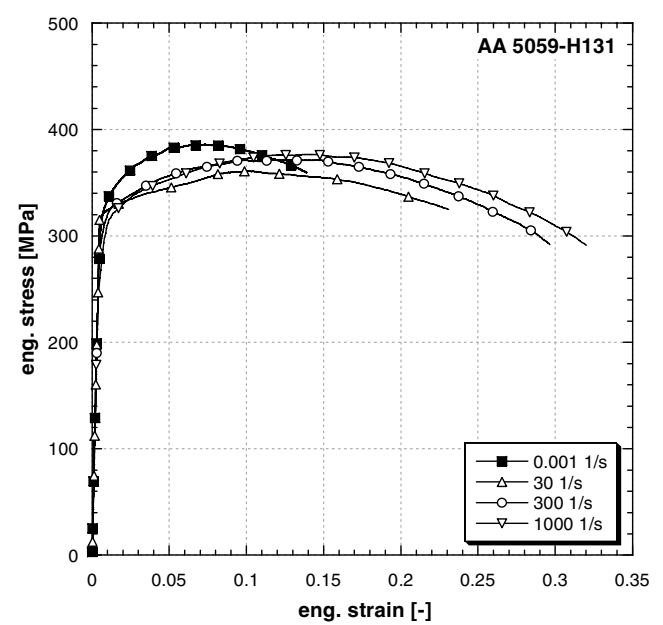

Fig. 6. Engineering stress versus strain at different strain-rates for AA 5059-H131.

Post-mortem examination of the specimens has also been made after fracture by measuring the diameter and the meridional radius of curvature at the reduced section for AA 5059-H131 and AA 7039-T651 respectively (as shown in figures 11 and 12). These data are used for the last point of the true stress-strain diagram by the Bridgman formula [7].

\section{Constitutive model}

Numerical simulations are widely used in order to predict the behaviour of structures at high strain rates.

Several material constitutive relationships describing the mechanical response of metallic materials at impact rates of strain are proposed in literature as for example Johnson-Cook relationship [8], here chosen for this investigation.

In this preliminary phase only isotropic hardening and strain-rates hardening have been considered, due to the 


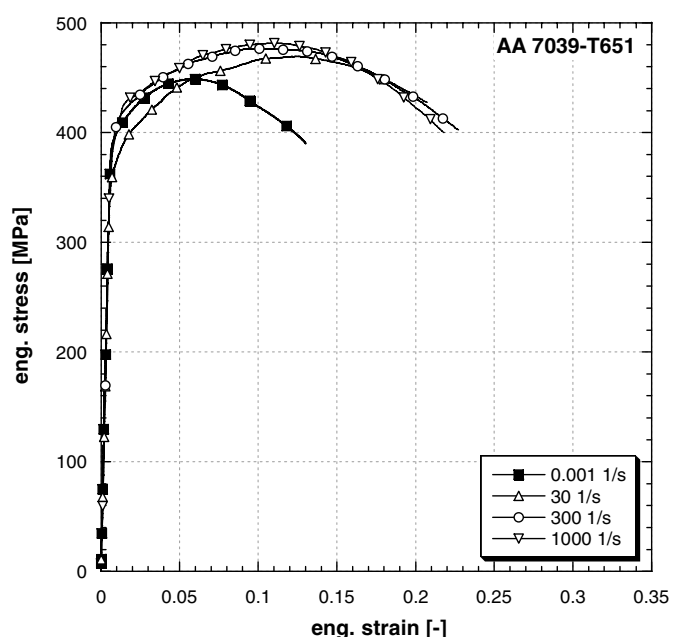

Fig. 7. Engineering stress versus strain at different strain-rates for AA 7039-T651.

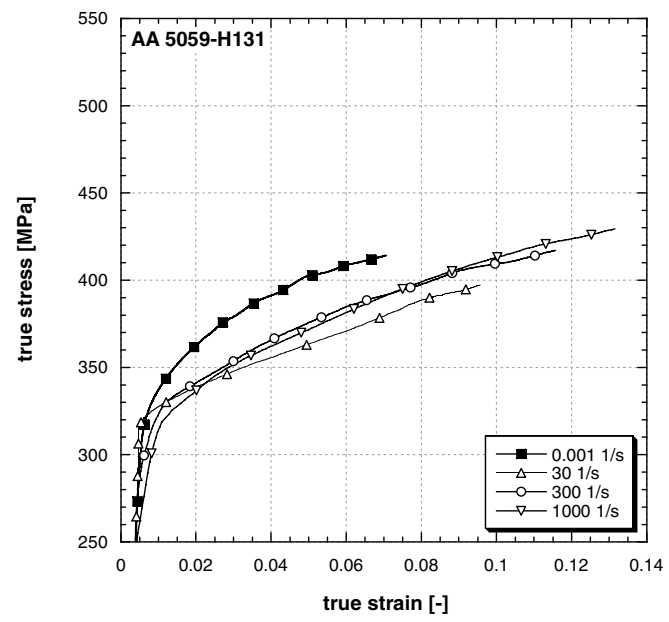

Fig. 8. True stress versus true strain at different strain-rates for AA 5059-H131.

unavailability of temperature data. The obtained parameters are shown in the next table 3, where the $c$ parameter is assumed as a mean value between tests at different strain-rates.

In order to show the goodness of the considered constitutive relation, a comparison amongst the Johnson-Cook model, the quasi-static and the $300 \mathrm{~s}^{-1}$ experimental data for AA 5059-H131 is reported in figure 13 while in figure 14 the same comparison is done for AA 7039-T651.

\section{Concluding remarks}

This first experimental research was conducted in order to study the strain-rate sensitivity of these two considered aluminium alloys.

A good repeatability of tests at different strain-rates was noted.

With respect to the obtained data it is possible to point out that AA 5059-H131 has shown a negative sensitivity to strain rate, a moderate strain-rate sensitivity of uniform

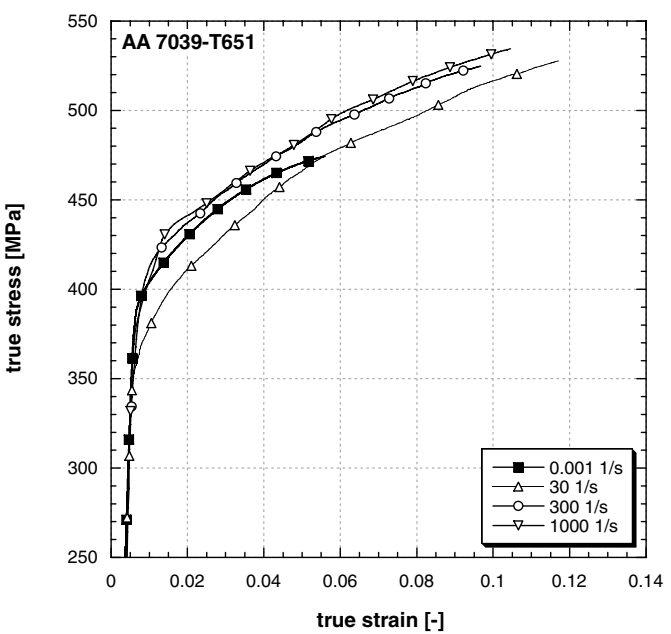

Fig. 9. True stress versus true strain at different strain-rates for AA 7039-T651.
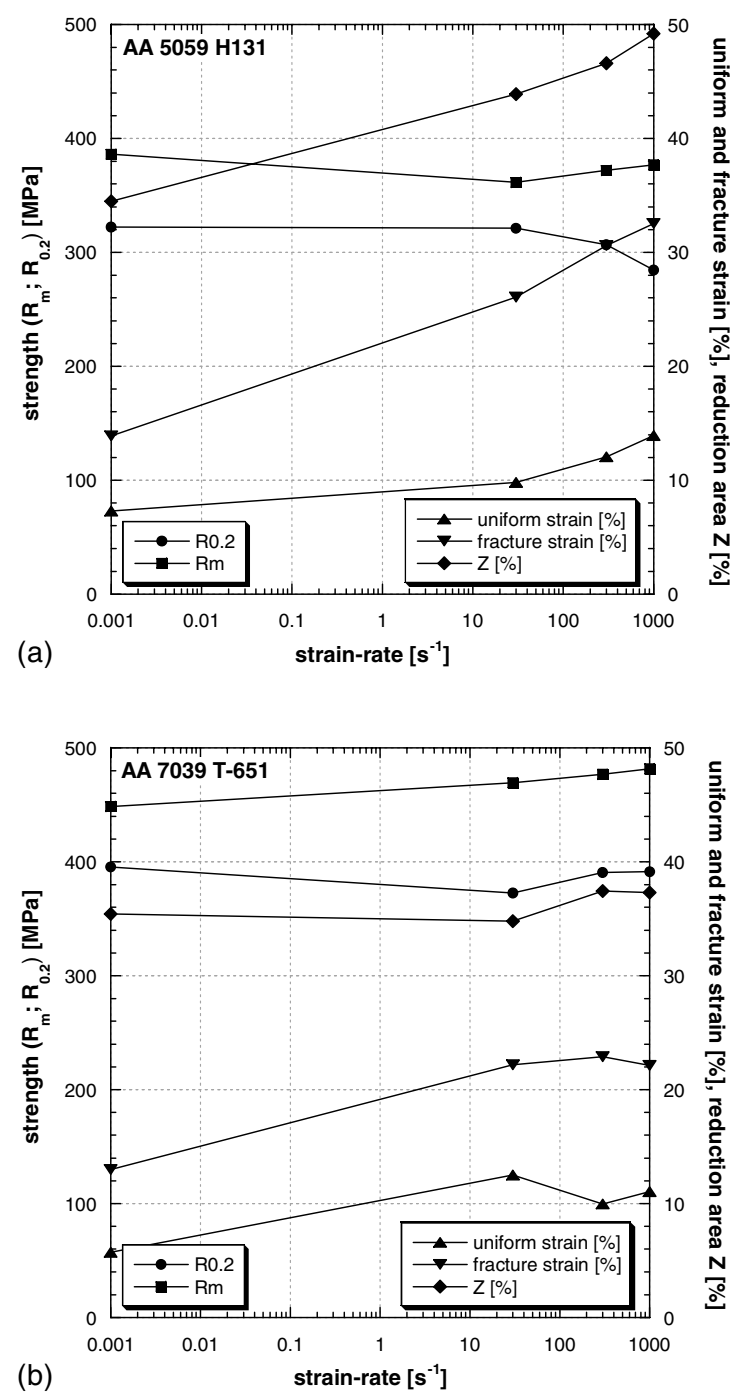

Fig. 10. (a) Trend of principal mechanical characteristics in function of the strain-rate for AA 5059-H131. (b) Trend of principal mechanical characteristics in function of the strain-rate for 7039-T651. 


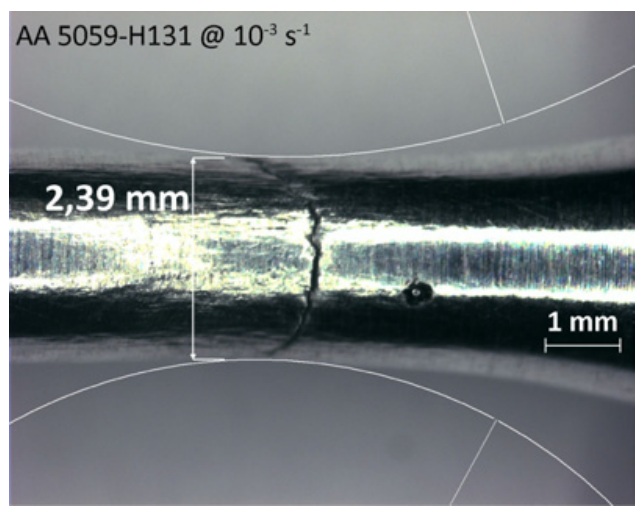

(a)

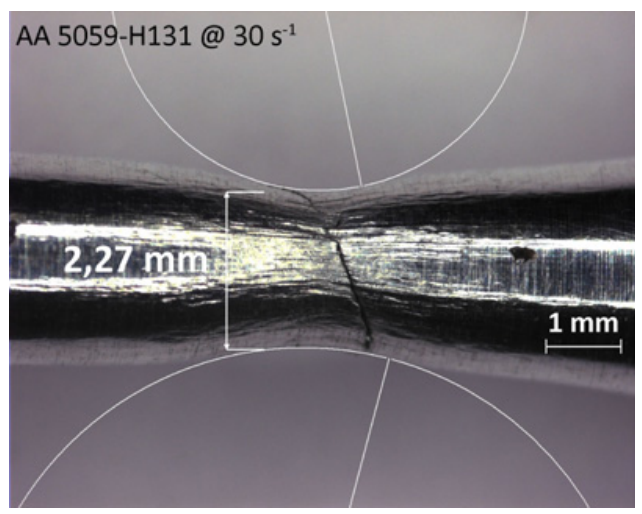

(b)

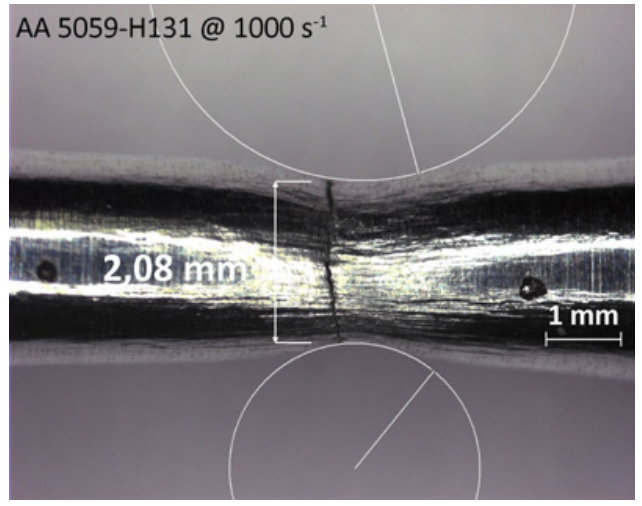

(c)

Fig. 11. (a) Sample AA 5059-H131 (\#01) @ 0,001 s-1. (b) Sample AA 5059-H131 (\#09) @ 30 s ${ }^{-1}$. (c) Sample AA 5059H131(\#03) @ 1000 s ${ }^{-1}$.

and fracture strain and a strong reduction of cross-sectional area at fracture.

On the other hand, the AA 7039-T651 has shown a negative and a positive strain-rate sensitivity at medium and high strain-rates respectively. A moderate strain-rate sensitivity of uniform and fracture strain was observed. Lastly, increasing the strain-rate, also a poor reduction of cross-sectional area at fracture was achieved.

The strain-rate dependence on ductility can be described by the cross-sectional area reduction of the necked region after fracture. The AA 5059-H131 shows an important increase in ductility, while the AA 7039-T651 shows

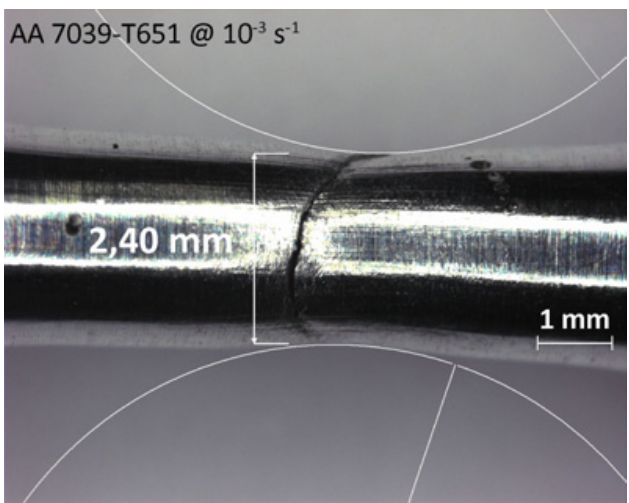

(a)

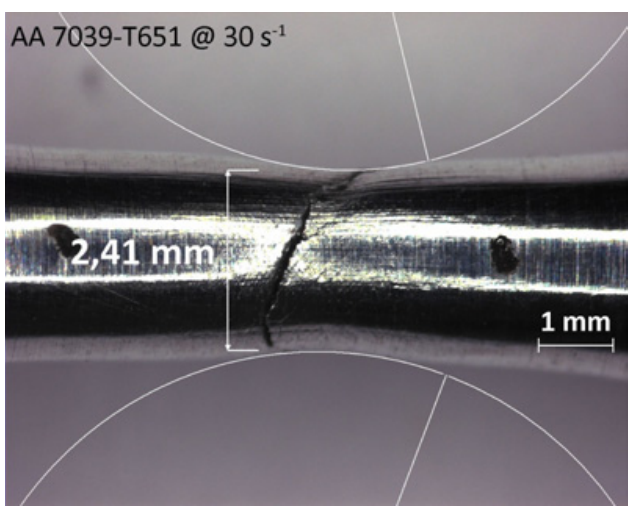

(b)

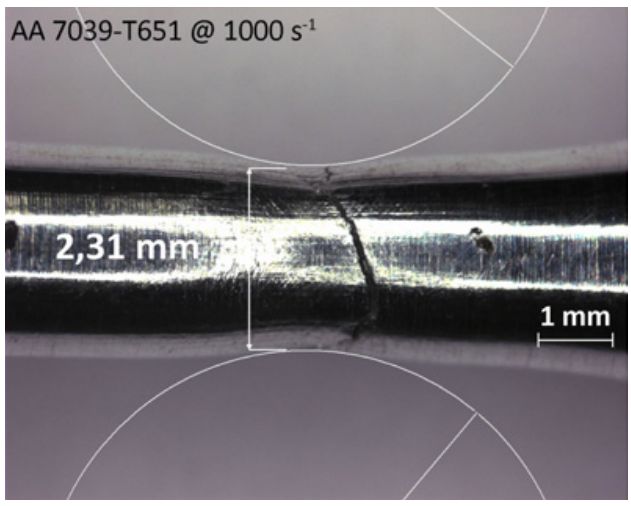

(c)

Fig. 12. (a) Sample AA 7039-T651 (\#01) @ 0,001 s $\mathrm{s}^{-1}$. (b) Sample AA 7039-T651 (\#09) @ 30 s-1. (c) Sample AA 7039T651(\#04)@1000 s ${ }^{-1}$.

Table 3. Johnson-Cook parameters for the analysed materials.

\begin{tabular}{|c|c|c|c|c|}
\hline & $\begin{array}{c}A \\
{[\mathrm{MPa}]}\end{array}$ & $\begin{array}{c}B \\
{[\mathrm{MPa}]}\end{array}$ & $\begin{array}{c}n \\
{[-]}\end{array}$ & $\begin{array}{c}c_{\text {mean }} \\
{[-]}\end{array}$ \\
\hline AA 5059-H131 & 321,1 & 412,8 & 0,5257 & $-0,0149$ \\
\hline AA 7039-T651 & 391,7 & 538,3 & 0,6015 & $+0,0010$ \\
\hline
\end{tabular}

a slight loss in ductility as strain-rate increase from quasistatic to medium, and a small increase in ductility at high strain-rate.

Regarding the Johnson-Cook model it is possible to point out that, even if the strain-hardening is extremely 


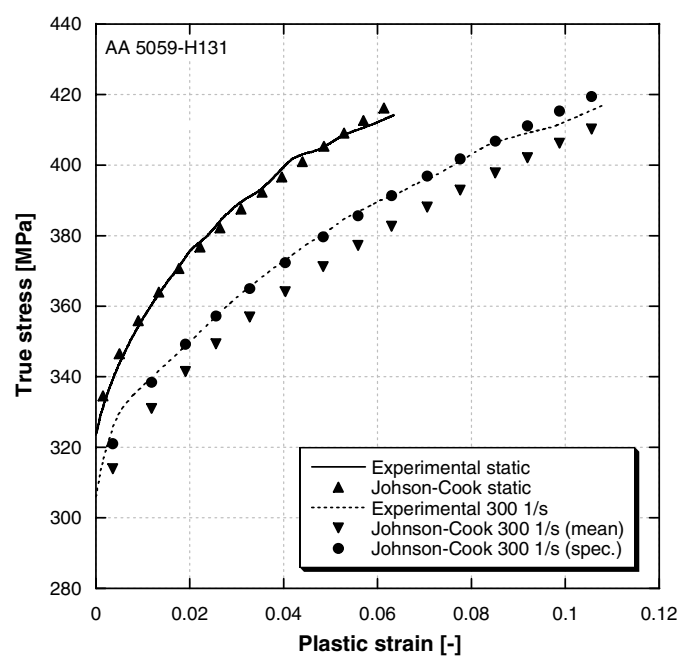

Fig. 13. Johnson-Cook fit for AA 5059-H131.

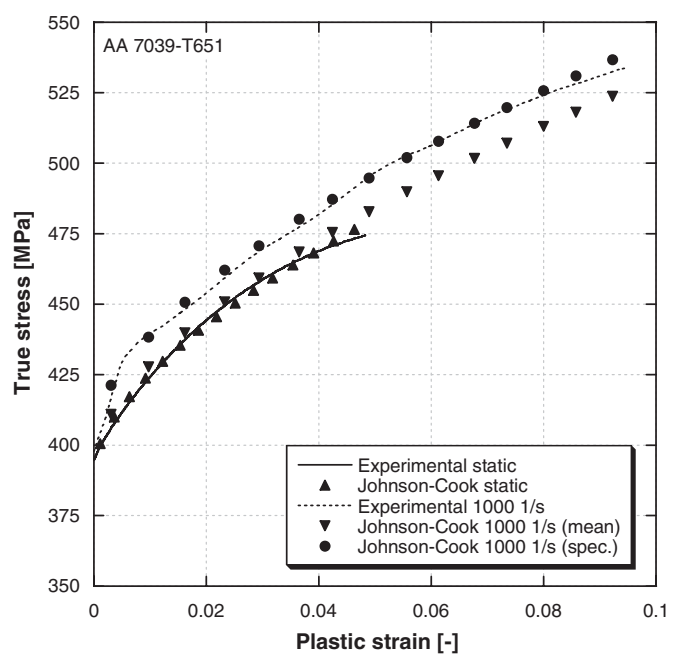

Fig. 14. Johnson-Cook fit for AA 7039-T651.

well fitted, the strain-rate sensitivity doesn't fit very well. This is probably due to the use of a mean value of the $c$ parameter. In order to explain this fact, in figures 13 and 14 are reported two versions of Johnson-Cook fit: (a), with the use of a mean value of $c$, and (b) with the use of a $c$ value specifically evaluated only for $300 \mathrm{~s}^{-1}$ tests.

The parameters of this constitutive relation, which include the strain-rate effect, can be easily used in the numerical simulations of dynamic events, in order to help the numerical community to perform design optimisation and safe design of both components and structures subjected to impulsive loads.

\section{Acknowledgements}

The Authors would like to acknowledge Aleris Aluminium Koblenz $\mathrm{GmbH}$ for providing the materials.

\section{References}

1. M.J. Handianfard, R. Smerd, S. Winkler and M. Worswick, Mater. Sci. Eng. A., 492 (2008), 283292.

2. E. Cadoni, M. Dotta, D. Forni and S. Bianchi, Applied Mechanics and Materials, 82 (2011), 124-129.

3. T. Naka, F. Yoshida, J. Mater. Process. Technol. 19 (1999), 89-90.

4. S.J. Pérez-Bergquist et al., Mater. Sci. Eng. A., 528 (2008), 283-292.

5. C. Albertini, M. Montagnani, Testing techniques based on the split Hopkinson bar, Institute of Physics Conference series, 21 (1974), 22-32.

6. C. Albertini, M. Montagnani, Journal NED 37, (1976), 115-124.

7. P.W. Bridgman, Studies in large plastic flow and fracture, Mc Graw-Hill, 1952.

8. Johnson, G.J., Cook, W.H. Proceedings of the Seventh International Symposium on Ballistics, The Hague, 1983, pp. 541-547. 